\title{
A Visual Sensor Network for Parking Lot Occupancy Detection in Smart Cities
}

\author{
Luca Baroffio, Luca Bondi, Matteo Cesana, Alessandro Enrico Redondi, Marco Tagliasacchi \\ Dipartimento di Elettronica, Informazione e Bioingegneria \\ Politecnico di Milano \\ Milano, Italy \\ \{name.surname\}@polimi.it
}

\begin{abstract}
Technology is quickly revolutionizing our everyday lives, helping us to perform complex tasks. The Internet of Things (IoT) paradigm is getting more and more popular and is key to the development of Smart Cities. Among all the applications of IoT in the context of Smart Cities, realtime parking lot occupancy detection recently gained a lot of attention. Solutions based on computer vision yield good performance in terms of accuracy and are deployable on top of visual sensor networks. Since the problem of detecting vacant parking lots is usually distributed over multiple cameras, adhoc algorithms for content acquisition and transmission are to be devised. A traditional paradigm consists in acquiring and encoding images or videos and transmitting them to a central controller, which is responsible for analyzing such content. A novel paradigm, which moves part of the analysis to sensing devices, is quickly becoming popular. We propose a system for distributed parking lot occupancy detection based on the latter paradigm, showing that onboard analysis and transmission of simple features yield better performance with respect to the traditional paradigm in terms of the overall rate-energyaccuracy performance.
\end{abstract}

Keywords-Parking Lot Occupancy Detection, Smart Parking, IoT, Visual Sensor Network, Visual Features

\section{INTRODUCTION}

Stimulated by recent findings in several research areas and off-the-shelf commercial products, the Internet of Things (IoT) paradigm is quickly becoming a reality in everyday life. Low-power and cheap microcontrollers and transceivers for digital communications, coupled with a great variety of sensors for the acquisition, transmission and analysis of different kinds of data are enabling users to access several applications in many domains, such as home automation, mobile healthcare, smart energy management and many others. The IoT paradigm fits particularly well in urban scenarios and is foreseen as a key technology for the realization of the Smart City concept. By optimizing the management and accessibility of many traditional public services (transport, lighting, surveillance, maintenance of public areas, etc.), future Smart Cities aim to increase the quality of life of citizens, while reducing the operational costs for public administrations [1].

Among all the applications of IoT in the context of Smart Cities, parking lot monitoring (also known as smart parking) has recently gained a lot of attentions from both the scientific community and the industry [2]. By combining data from parking lots with web based services and intelligent displays, smart parking allows drivers to find vacant parking lots near their destinations quickly and easily. This provides several benefits, such as fewer $\mathrm{CO}^{2}$ emissions from cars, reduced traffic congestion and finally, less stressed and happier citizens.

Clearly, the parking lot occupancy information (i.e., knowing which parking lots are vacant) plays a crucial role in such a scenario. In the last few years, several solutions have been proposed for solving such a problem: each solution differs in the type of sensors used to get the occupancy information (e.g., magnetometers, light sensors, microphones, etc.), in the communication technology (e.g., wired / wireless) and in the physical deployment (e.g., attached to the ceilings, buried under the asphalt, etc.). Of particular interest are those solutions based on Wireless Sensor Networks (WSN), characterized on the one hand by low installation and management costs, and on the other hand by a great flexibility in supporting different sensors that can accurately keep track of parked vehicles.

Recently, WSNs have been enabled with vision capabilities, giving rise to the so-called Visual Sensor Networks (VSNs). In VSNs some nodes are geared with cameras, thus being able to acquire, process and transmit images and video from the environment. VSNs constitute a natural choice for the scenario of parking lot monitoring: a single camera node may acquire an image covering several parking lots, compress it using a suitable encoder (e.g., JPEG) and transmit it to a central controller, where an image processing algorithm is used extract the occupancy information. However, such a compress-then-analyze (CTA) paradigm generally suffers from the problem of generating large amount of multimedia data, resulting in high energy consumption and bandwidth usage, which are generally constrained in VSN applications. To overcome this problem, a paradigm shift has emerged recently. In the analyze-then-compress (ATC) paradigm, the visual content is processed locally at the camera nodes to extract local visual features, which are then transmitted to the central controller for analysis. Such feature-based representation can be much more compact than the pixelbased one, thus making the ATC paradigm a preferable choice [3], [4].

In this paper, we propose a parking lot occupancy detec- 
tion system based on a VSN that can be operated using the ATC paradigm. Camera nodes extract local features specifically designed for the task at hand and transmit them to a central controller where a classifier is used to retrieve the occupancy information. We implement the system on a real VSN testbed, and evaluate its accuracy and energy performance, also considering the traditional CTA paradigm as benchmark.

The rest of the paper is organized as follows: Section II summarizes the related work in the area of smart parking and Section III details the parking lot monitoring system. Section IV reports a thorough experimental evaluation of the proposed system and Section V concludes the paper.

\section{RELATED WORK}

Several works in the past addressed the problem of implementing reliable Parking Guidance and Information Systems (PGIS), by locating vacant spaces in parking lots and disseminating such information to drivers. The existing works can be broadly categorized in two areas, depending on whether or not the particular solution uses cameras to infer the location of free parking lots.

\section{A. WSN-based systems}

Many systems are implemented by relying on a network of sensor nodes, which are generally buried under the asphalt so that each node senses one parking space. Different types of sensor have been used in the past: the works in [5] and [6] use light sensors and evaluate the performance of the system using remote controlled toy cars. In [7], the authors leverage a WSN composed of optical sensors, while in [8] a combination of magnetic and ultrasonic sensors is used. A comparison of the performance of different sensors for smart parking can be found in [9].

\section{B. Camera-based systems}

One of the main drawbacks of WSN-based systems is scalability, as installing and managing one sensor per parking space may be cumbersome and expensive. To overcome this issue, vision-based systems can be used: each camera can cover more than one parking space thus providing a scalable solution. In [10], a parking space finder application is presented: webcams are utilized for retrieving the availability of parking spaces near a users destination. However, neither details on the algorithm used to extract such information nor its accuracy are reported. The work in [11] uses enhanced background subtraction techniques to infer the status of each parking space, while in [12] a fuzzy $c$-means (FCM) clustering algorithm is proposed and compared against a SVM classifier, using PCA basis vectors extracted from vacant or occupied parking lot images to train the classifiers. The authors report an accuracy of more than 99\% in an indoor scenario. In [13], a rich parking image dataset is presented and made available to help researchers

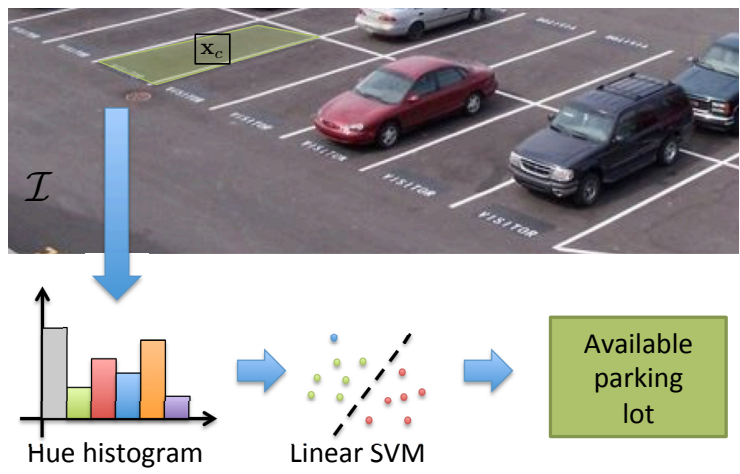

Figure 1. A region around the center of the parking lot is extracted, and a feature vector is computed by means of a histogram of pixel hue values. A linear SVM is employed to classify each parking lot as either vacant or occupied.

in testing and comparing different algorithms for parking space classification. Moreover, the authors present a texturalbased classifiers based on Local Binary Patterns (LBP) that achieves excellent performance in outdoor scenarios.

Finally, several recently released commercial products leverage vision techniques in order to help drivers finding available parking lots [14][15].

\section{PRoposed System}

The proposed system is composed of a network of wireless camera nodes running a parking lot occupancy detection algorithm. The following sections provide details on the particular algorithm used and on the software/hardware implementation of the system.

\section{A. Parking space classification}

With reference to Figure 1, let $\mathcal{I}$ be the image of the parking lot acquired by a camera node, containing $C$ parking spaces. Since camera nodes are likely to be deployed statically in the parking lot, we assume to know a-priori, for each parking space, the pixel coordinates of its center $\mathbf{x}_{c}$, together with its width $w_{c}$ and height $h_{c}$, with $c=1 \ldots C$. For each parking space, a squared subregion of size $\min \left(w_{c}, h_{c}\right)$ centered in $\mathbf{x}_{c}$ is extracted from the image. Each subregion is converted from RGB to HSV colorspace, and the hue component is used to populate a histogram $\mathbf{h}_{c}$. The histograms are used as local features to determine whether or not a parking space is vacant. For the task at hand, we trained a linear SVM classifier with regularization constant $K=100$. The classifier is fed with normalized hue-histograms extracted from labeled data both in training and in test phase.

\section{B. Testbed realization}

The classification algorithm has been implemented on a real visual sensor network testbed based on BeagleBone camera nodes operated with Debian Linux [16]. In particular, 


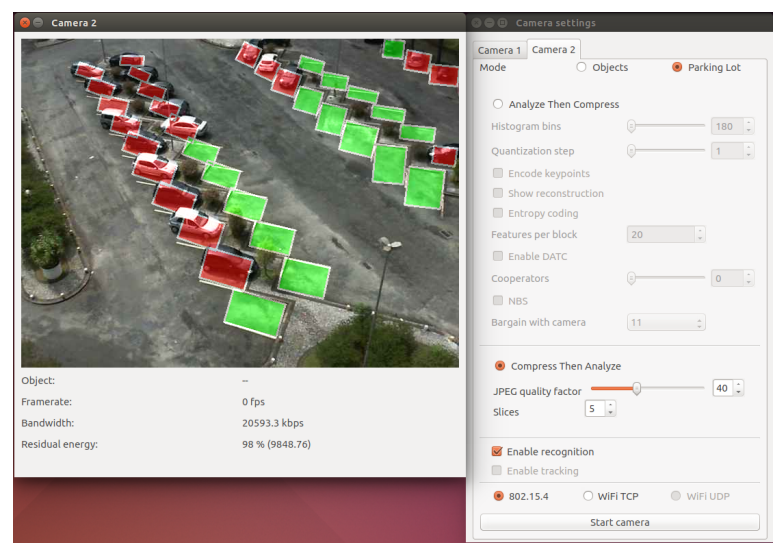

(a)

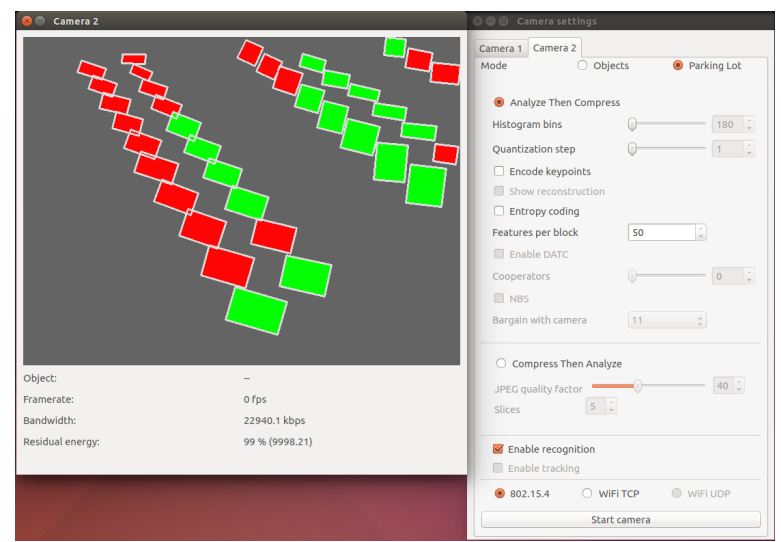

(b)

Figure 2. A screen-shot of the testbed GUI for (a) the CTA case and (b) the ATC case. Note that the ATC paradigm completely disregards the transmission of a compressed image, still allowing occupancy detection to be performed correctly.

each camera node is composed of a $1 \mathrm{GHz}$ battery-operated BeagleBone Black, coupled with a low-cost VGA camera and an IEEE 802.15.4 wireless transceiver. A laptop with IEEE 802.15.4 communication capabilities is used as central controller and runs a Graphical User Interface (GUI) to control in real time the operation of camera nodes in the network. Each camera can be operated using the CTA or ATC paradigms, as illustrated in Figures 2(a) and 2(b). In CTA, JPEG compressed images are transmitted from the camera to the central controller. Here, the features described in Section III-A are extracted and used to get the parking lot occupancy information. From the GUI, a user can change in real-time the JPEG quality factor and in turn the amount of data which is generated by cameras.

Conversely, if the ATC paradigm is selected, camera nodes extract parking features directly from the acquired images and transmit them to the central controller. From the GUI it is possible to change the number of bins to use in each hue histogram, together with the number of bits to be used to represent the number of occurrences in each bin. Again, such settings are meant to control the amount of data generated by camera nodes. Note also that the availability of the features at the central controller allows for the implementation of advanced classification algorithm, e.g., by fusing features from multiple views.

Clearly, regardless of the particular paradigm chosen (i.e., CTA or ATC), there is a trade-off between the amount of data generated by cameras and the accuracy of the parking space classification. The following section provides a detailed experimental evaluation of the proposed system.

\section{EXPERIMENTAL RESULTS}

We are interested in assessing the accuracy of the parking space classifier when varying both the Visual Sensor Network operational paradigm (CTA or ATC) and the paradigmspecific parameters. As explained in Section III-B, for both

\begin{tabular}{|lccc|}
\hline Dataset & Num. of parking lots & Training samples & Test samples \\
\hline \hline UFPR04 & 28 & 54.236 & 51.912 \\
\hline UFPR05 & 37 & 86.600 & 79.480 \\
\hline PUCPR & 100 & 224.400 & 222.900 \\
\hline
\end{tabular}

Table I

CHARACTERISTICS OF THE USED DATASETS.

paradigms it is possible to modify the amount of data generated and transmitted by camera nodes to the central controller. On the one hand, this directly impacts on the bandwidth usage and energy consumption of camera nodes: since they are the major limitations in visual sensor networks, it is imperative to keep them low. On the other hand, decreasing the JPEG image quality in CTA or the histogram resolution in ATC affects the classifier accuracy. To analyze the relationship between bandwidth usage, energy consumption and classifier accuracy, we performed several experiments using the visual sensor network testbed we have implemented. Instead of testing the VSN in a real scenario, we relied on the Pk-lot dataset provided in [13], which contain images of parking lots under different weather conditions (overcast, sunny and rainy periods), organized in three different datasets (UFPR04, UFPR05 and PUCPR). Each image in the datasets is annotated with information on the free/occupied parking lots and each one of the three dataset is divided in a training and testing set. We used the training set to train the SVM classifier, and all experiments were then performed on the testing sets. Table I reports the specifications for each dataset.

We pre-loaded images from the datasets on the camera nodes after resizing them to VGA resolution. This allowed to perform a great number of experiments in a controlled environment, changing each time the operational parameters through the GUI. In particular, we varied the JPEG quality factor in CTA in the range $\{7,10,20,30,40\}$. For ATC, we 


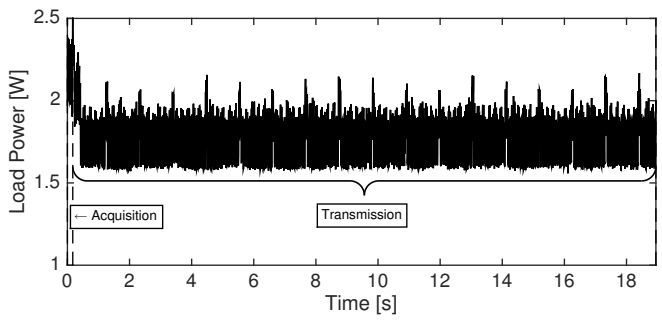

(a)

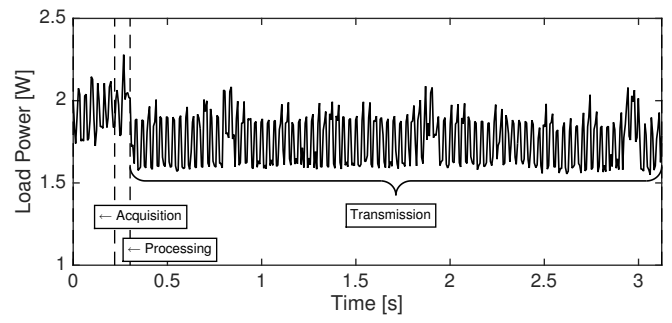

(b)

Figure 3. Power consumption of the proposed VSN for parking monitoring in CTA mode (a) and ATC mode (b) at a target application accuracy of $90 \%$. For CTA, this corresponds to a JPEG quality factor equal to 20 . The same accuracy is reached in ATC with the transmission of a 45 bins histogram for each parking lot, greatly reducing the transmission time and the corresponding overall energy consumption.

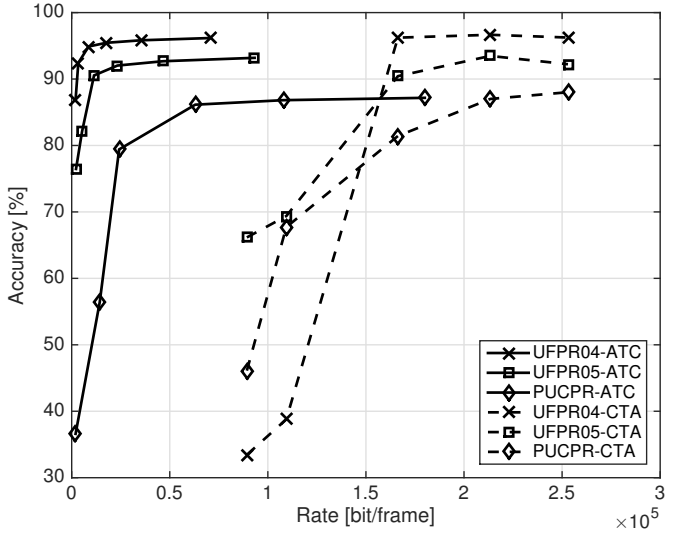

Figure 4. Accuracy vs Rate in ATC and CTA mode. The rate is the amount of data sent from the camera node to the sink node for a single shot. For the ATC case 5, 11, 22, 45, 90 and 180 bins are used. For the CTA case JPEG quality factor is set to $7,10,20,30$ and 40 .

varied the number of bins in each hue histogram in the range $\{5,11,22,45,90,180\}$.

For each experiment, we kept track of (i) the accuracy of the parking classifier, computed as the fraction of correctly classified parking lots over the total number of parking lots in the evaluation dataset, (ii) the average amount of data generated by camera nodes and (iii) the corresponding camera node energy consumption. In the latter case, we used an Adafruit INA219 current sensor to measure the instantaneous current consumption of the camera node, as illustrated in Figures 3(a) and 3(b). Figures 4 and 5 show the rate-accuracy and energy-accuracy performance of the proposed system, respectively. Several considerations can be made from the inspection of such figures:

1) A clear trade-off between rate and accuracy of classification is present. For both paradigms, increasing the rate produced by camera nodes increases the classification accuracy (Figure 4). In CTA, using a low JPEG QF greatly affects the accuracy of classification. This is not true for ATC, where using a limited number of bins affects only partially the classification accuracy.

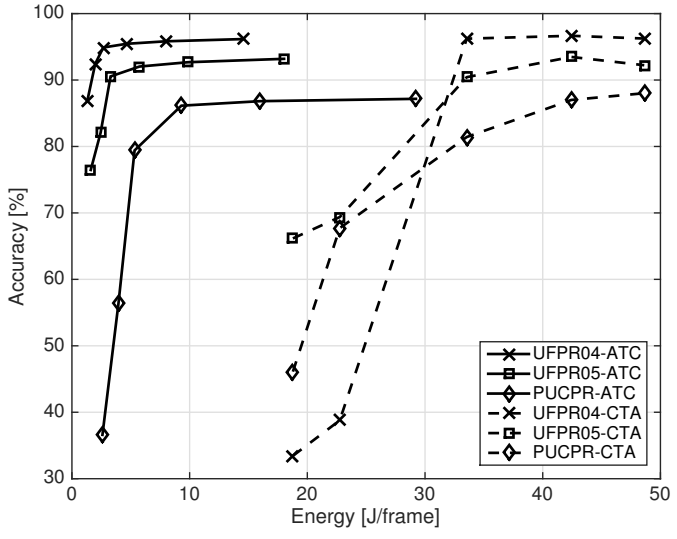

Figure 5. Accuracy vs Energy in ATC and CTA mode. The energy is measured integrating power over time during acquisition, extraction (ATC only), coding and transmission phases. The same parameters as in Figure 4 are used.

2) The ATC paradigm outperforms the traditional CTA paradigm from both the bandwidth usage and energy consumption perspective. From Figure 4 , it is clear that for the same target accuracy, ATC requires approximately one half of the CTA bandwidth. This result can be also improved by adding an arithmetic encoder to the ATC pipeline just before the transmission stage. This is left to future investigation.

3) Figure 5 shows that for a single VGA shot the ATC paradigm suits better battery operated devices due to the significantly lower energy consumption per shot. Even without encoding, the energy consumption for a single frame in ATC mode is just one quarter of the one consumed in CTA, at the same accuracy level.

4) Figure 3(a) clearly shows that transmitting a JPEGencoded VGA image over a 802.15.4 communication channel is an expensive operation in terms of energy consumption and it introduces a significant amount of latency. On the contrary, the ATC approach 3(b) is more than 7 times faster due to the light features extraction algorithm explained above, together with 
the very limited amount of data being sent to the sink node.

5) Overall, the proposed classification algorithm allows to obtain very good accuracy values, as high as 96, 93 and $87 \%$ on UFPR04, UFPR05, PUCPR datasets, respectively.

\section{CONCLUSIONS}

We presented a system for distributed parking lot occupancy detection implemented on top of a low-power Visual Sensor Network and simple visual features. Such system is operated according to the analyze-then-compress (ATC) paradigm, that requires features to be extracted by sensing devices, encoded, and transmitted to a central unit for further processing. The system can be operated in realtime resorting to multiple camera nodes and allows for a great degree of flexibility. We validate the effectiveness of the system investigating the tradeoff between accuracy, transmission bandwidth and energy requirements for the task at hand, comparing our system to a baseline method based on the transmission of compressed images and centralized visual analysis. Tests show the superiority of our approach, that yields good performance while having low requirements in terms of energy and bitrate and achieving low task latency. Future work will include devising more effective visual features and coding methods and validating the effectiveness of the method in a real deployment.

\section{ACKNOWLEDGMENT}

This work has been supported by the Future and Emerging Technologies (FET) programme within the Seventh Framework Programme for Research of the European Commission, under FET-Open project GreenEyes (grant number: 296676), and by the Italian Ministry for Education, University and Research (MIUR) through the national cluster project SHELL, Smart Living technologies (grant number: CTN01_00128_111357).

\section{REFERENCES}

[1] A. Zanella, N. Bui, A. Castellani, L. Vangelista, and M. Zorzi, "Internet of things for smart cities," Internet of Things Journal, IEEE, vol. 1, no. 1, pp. 22-32, Feb 2014.

[2] L. Sanchez, L. Munoz, J. A. Galache, P. Sotres, J. R. Santana, V. Gutierrez, R. Ramdhany, A. Gluhak, S. Krco, E. Theodoridis, and D. Pfisterer, "Smartsantander: Iot experimentation over a smart city testbed," Computer Networks, vol. 61, no. 0, pp. $217-238,2014$, special issue on Future Internet Testbeds - Part I.

[3] L. Baroffio, M. Cesana, A. Redondi, and M. Tagliasacchi, "Performance evaluation of object recognition tasks in visual sensor networks," in Teletraffic Congress (ITC), 2014 26th International, Sept 2014, pp. 1-9.
[4] - "Compress-then-analyze vs. analyse-then-compress: Two paradigms for image analysis in visual sensor networks," in IEEE International Workshop on Multimedia Signal Processing (MMSP) 2013, Pula, Italy, September 2013.

[5] V. Tang, Y. Zheng, and J. Cao, "An intelligent car park management system based on wireless sensor networks," in Pervasive Computing and Applications, 2006 1st International Symposium on, Aug 2006, pp. 65-70.

[6] S. Srikanth, P. Pramod, K. Dileep, S. Tapas, M. Patil, and C. Sarat, "Design and implementation of a prototype smart parking (spark) system using wireless sensor networks," in Advanced Information Networking and Applications Workshops, 2009. WAINA '09. International Conference on, May 2009, pp. 401-406.

[7] J. Chinrungrueng, U. Sunantachaikul, and S. Triamlumlerd, "Smart parking: An application of optical wireless sensor network," in Applications and the Internet Workshops, 2007. SAINT Workshops 2007. International Symposium on, Jan 2007, pp. 66-66.

[8] S. Lee, D. Yoon, and A. Ghosh, "Intelligent parking lot application using wireless sensor networks," in Collaborative Technologies and Systems, 2008. CTS 2008. International Symposium on, May 2008, pp. 48-57.

[9] R. Kumar, N. Chilamkurti, and B. Soh, "A comparative study of different sensors for smart car park management," in Intelligent Pervasive Computing, 2007. IPC. The 2007 International Conference on, Oct 2007, pp. 499-502.

[10] S. Nath, A. Deshpande, Y. Ke, P. B. Gibbons, B. Karp, and S. Seshan, "Irisnet: An architecture for internet-scale sensing services," in $V L D B, 2003$, pp. 1137-1140. [Online]. Available: http://www.vldb.org/conf/2003/papers/S37P07.pdf

[11] S.-F. Lin, Y.-Y. Chen, and S.-C. Liu, "A vision-based parking lot management system," in Systems, Man and Cybernetics, 2006. SMC '06. IEEE International Conference on, vol. 4, Oct 2006, pp. 2897-2902.

[12] H. Ichihashi, T. Katada, M. Fujiyoshi, A. Notsu, and K. Honda, "Improvement in the performance of camera based vehicle detector for parking lot," in Fuzzy Systems (FUZZ), 2010 IEEE International Conference on, July 2010, pp. 1-7.

[13] P. R. de Almeida, L. S. Oliveira, A. S. B. Jr., E. J. S. Jr., and A. L. Koerich, "Pklot - a robust dataset for parking lot classification," Expert Systems with Applications, vol. 42, no. 11, pp. 4937 - 4949, 2015.

[14] "Parkit - the x-ray for parking lots," http://parkitfaster.com.

[15] “Parquery," http://www.parquery.com.

[16] L. Baroffio, A. Canclini, M. Cesana A.Redondi, M. Tagliasacchi, G. Dan, E. Eriksson, V. Fodor, J. Ascenso, and P. Monteiro, "Enabling visual analysis in wireless sensor networks," in Image Processing (ICIP), 2014 IEEE International Conference on, Oct 2014, pp. 3408-3410. 